\title{
Splitstrains, a tool to identify and separate mixed Mycobacterium tuberculosis infections from WGS data
}

\author{
Einar Gabbassov ${ }^{1,2, *}$, Miguel Moreno-Molina ${ }^{3}$, Iñaki Comas ${ }^{3}$, Maxwell Libbrecht ${ }^{1}$ and Leonid Chindelevitch ${ }^{4, *}$
}

\begin{abstract}
The occurrence of multiple strains of a bacterial pathogen such as M. tuberculosis or $\mathrm{C}$. difficile within a single human host, referred to as a mixed infection, has important implications for both healthcare and public health. However, methods for detecting it, and especially determining the proportion and identities of the underlying strains, from WGS (whole-genome sequencing) data, have been limited. In this paper we introduce Splitstrains, a novel method for addressing these challenges. Grounded in a rigorous statistical model, Splitstrains not only demonstrates superior performance in proportion estimation to other existing methods on both simulated as well as real M. tuberculosis data, but also successfully determines the identity of the underlying strains. We conclude that Splitstrains is a powerful addition to the existing toolkit of analytical methods for data coming from bacterial pathogens and holds the promise of enabling previously inaccessible conclusions to be drawn in the realm of public health microbiology.
\end{abstract}

\section{DATA SUMMARY}

The authors confirm all supporting data, code and protocols have been provided within the article or through supplementary data files.

Supplementary data files can be found at 10.6084/ m9.figshare.14562321.

\section{INTRODUCTION}

Bacterial infections by pathogens such as Mycobacterium tuberculosis and Clostridium difficile often occur as mixed infections $[1,2]$, whereby a single patient is infected by several different strains of the same organism. Eukaryotic pathogens such as the main etiological agent of malaria, Plasmodium falciparum, can also cause mixed infections [3]. The identification of such mixed infections can be important for reasons including both patient-level decisions [4] as well as public health measures [5]. In the latter setting, if the tracing of the origins of the mixed infection is needed, it may be additionally required to separate the mixed infection into its constituent strains. The separation may also be informative when the mixed infection is hetero-resistant [6], namely, when some, but not all, the strains are resistant to a particular antimicrobial drug. Moreover, a failure to identify the within-host pathogen diversity can lead to misdiagnosing a relapse and reinfection [7]. However, so far, the problem of identifying mixed infections and separating them into their constituent strains has not received a sufficient amount of attention in the literature.

Although older techniques based on the detection of specific regions, such as VNTR (variable-number tandem repeats) [8], are often able to detect such a mixed infection [9], this is not always the case with next-generation sequencing. The main challenge is that the presence of two alternative alleles in a given genomic position may signal a sequencing error as well as the presence of multiple strains. The key distinguishing feature of a mixed infection is the consistency of the fraction of the sample attributable to the sub-dominant strain across

Received 16 February 2021; Accepted 10 May 2021; Published 24 June 2021

Author affiliations: ${ }^{1}$ School of Computing Science, Simon Fraser University, Burnaby, BC, Canada; ${ }^{2}$ Department of Mathematics, Simon Fraser University, Burnaby, BC, Canada; ${ }^{3}$ Instituto de Biomedicina de Valencia, Valencia, Spain; ${ }^{4}$ MRC Centre for Global Infectious Disease Analysis, School of Public Health, Imperial College, London, UK.

*Correspondence: Leonid Chindelevitch, l.chindelevitch@imperial.ac.uk; Einar Gabbassov, egabbass@sfu.ca

Keywords: hetero-resistance; public health microbiology; maximum likelihood; mixed infection; Mycobacterium tuberculosis; multiple-strain infection. Abbreviations: AUC, area under curve; BAM, binary sequence alignment map; FPR, false positive rate; GFF, generic feature format; GMM, gaussian mixture model; NCBI, National Center for Biotechnology Information; RMSE, root mean square error; ROC, receiver operating characteristic; SNP, single-nucleotide polymorphism; TPR, true positive rate; VCF, variant call format; VNTR, variable-number tandem repeats; WGS, whole genome sequencing.

Repository for the source code for SplitStrains: https://github.com/WGS-TB/SplitStrains.

Data statement: All supporting data, code and protocols have been provided within the article or through supplementary data files. One supplementary table is available with the online version of this article.

000607 (2021 The Authors 
most of the variable positions. Thus, depending on the depth of coverage, the similarity between the constituent strains and the proportions in which they are mixed, the problem of detecting and separating mixed strains may vary from straightforward to nearly infeasible.

Several methods for this problem have appeared over the past decade. Eyre et al. [2] propose a Mixed Infection Estimator, a two-step approach for mixture proportion estimation using a maximum likelihood analysis and mixed strain identification using a custom database. Even though the paper presents results for $C$. difficile, the mixture estimation algorithm can be generalized to other pathogens such as $M$. tuberculosis. This method computes a deviance statistic and uses a threshold value for this statistic to detect mixed infections. As this algorithm was initially designed for C. difficile and relies on a custom database of sequences to identify the constituent strains, it could only be used for mixture proportion estimation in our context. More recently, Sobkowiak et al. [10] developed MixInfect, a method for mixture proportion estimation using a Bayesian model-based clustering technique. This method calculates the ratio of heterozygous calls to total SNPs (single nucleotide polymorphisms) and uses a threshold on this ratio to identify mixed samples. While this algorithm can estimate mixture proportions it does not provide any functionality for resolving the constituent strains. The most recent method, QuantTB by Anyansi et al. [11], relies on a specially constructed publicly available database of 2166 M. tuberculosis assemblies from NCBI [12]. This method provides mixture estimates of WGS samples as well as the identification of strains whose sequence is similar to the ones included in the database. To determine the constituent strains, this method compares the sample to the sequences in the reference database, scoring each of the assemblies. The algorithm then determines how many constituent strains are present in a sample. This approach does not generalize to situations where the underlying strains lack close representatives in the database, which makes its performance highly dependent on the database's representation of the common strains in the relevant local context.

In this paper, we address this problem with a tool called SplitStrains, grounded in a rigorous statistical framework. It is based on formulating, for a given set of WGS reads, two alternative hypotheses, namely: the reads belong to a single strain (null hypothesis) or to a mixture of two strains (alternative hypothesis). We then use the EM (Expectation-Maximization) algorithm [13] to estimate the parameters of both hypotheses, and compare their likelihoods to draw a conclusion. As a result, we simultaneously obtain:

- A call to decide whether the sample represents a single (pure) or a mixed infection,

- A likelihood ratio between the alternative and the null hypothesis for the call, and,

- If mixed, the proportion of each constituent strain and a Binary Sequence Alignment Map (BAM) file grouping the reads belonging to each constituent strain.

\section{Impact Statement}

When multiple strains of a pathogenic organism are present in a patient, it may be necessary to not only detect this, but also to identify the individual strains. However, this problem has not yet been solved for bacterial pathogens processed via whole-genome sequencing. In this paper, we propose the splitstrains algorithm for detecting multiple strains in a sample, identifying their proportions, and inferring their sequences, in the case of Mycobacterium tuberculosis. We test it on both simulated and real data, with encouraging results. We believe that our work opens new horizons in public health microbiology by allowing a more precise detection, identification and quantification of multiple infecting strains within a sample.

Our results on both simulated and real M. tuberculosis data show that SplitStrains is effective at identifying mixed infections and continues to perform well even at a relatively low depth of coverage $(60 \times)$ and low genetic distance (20 SNPs) between strains. Moreover, Splitstrains outperforms previously published tools Mixed infection estimator, MixInfect and QuantTB on simulated data. Furthermore, our results show that Splitstrains accurately separates the constituent strains provided that their proportions are not too close to each other and they are not too similar. SplitStrains is available on GitHub: https:// github.com/WGS-TB/SplitStrains.

\section{METHODS}

This part of the paper is organized as follows. First, we briefly describe the datasets used in our analysis. Second, we explain the construction of the feature vector used in our probabilistic model and show how to use it to classify an isolate. Third, we define the Naïve Bayes Classifier for the assignment of reads to strains. Lastly, we show how this approach can be generalized to three or more strains.

We begin by describing the datasets used in our analysis. We report the average number of SNPs relative to the reference genome in the Results section. Here we additionally report the average number of heterogeneous SNPs, defined by a $0 / 1$ in the GT field of the VCF file produced by aligning the sample to the reference genome. We note that the number of heterogeneous SNPs depends on the alignment and variantcalling steps of the pipeline. Therefore, for the in silico datasets, this number may be lower than the total number of SNPs added to the reference genome when generating the sample. We report the per-sample statistics in Table S1 (available in the online version of this article).

Dataset A, in vitro. The 48 mixed M. tuberculosis samples presented in [10] are artificially generated in vitro by combining the DNA from two clinical cultures of $M$. 
tuberculosis. The DNA is quantified through spectrophotometry in liquid culture and combined to produce four sets of 12 samples with major strain proportions of 70, 90, 95\% (mixed) and $100 \%$ (pure). The average number of heterogeneous SNPs is 327 .

Dataset B, in silico. The 60 artificial samples presented in [14] are generated from the standard reference genome for $M$. tuberculosis by substituting randomly chosen alleles at each of 553 genes in an essential core genome MLST scheme (ecgMLST), created by intersecting the set of core genes in an existing scheme with the set of 615 essential M. tuberculosis genes [15]. The full dataset contains three pure genomes, with ten samples generated from each one by varying the depth of coverage from 10 to 100 in increments of ten, and three mixed genomes obtained by mixing an additional three pure genomes in pairs, with ten samples generated from each by varying the major strain proportion from $50 \%$ to $95 \%$ in $5 \%$ increments. The average number of heterogeneous SNPs is 2843 .

Dataset C, in silico. For this dataset, generated specifically for this paper, the constituent strains are produced from the H37Rv reference genome. The WGS data is produced by the ART simulator [16] with the following settings:

(1) Profile: HiSeqX PCR free

(2) Read length: 150

(3) Per base sequence quality scores: 20 to 30 on the Sanger/ Illumina 1.9 scale.

(4) Quality shift: in order to match the quality of the real data, we shifted the quality scores down by nine to produce relatively uncertain sequences with high sequencing errors.

(5) Depth of coverage: 100 for single-strain and two-strain samples and 150 for three-strain samples.

The dataset consists of eight two-strain mixtures, six threestrain mixtures, and eight single strains. The two-strain mixtures have major strain proportions varying from $50 \%$ to $95 \%$ in $5 \%$ increments, with $55 \%$ and $60 \%$ omitted. The six three-strain samples have proportions 10:25:65, 15:30:55, 20:35:45, 25:40:35, 30:45:25, and 35:50:15. The average number of heterogeneous SNPs is 136 for the two-strain samples and 583 for the three-strain samples. The simulated reads were aligned back to the reference genome with BWA-MEM [17].

Dataset E, in silico. For this dataset, generated specifically for this paper, the constituent strains are produced from the same H37Rv reference genome with $N \in\{10,15,20$, $25\}$ random base substitutions. This yields four subsets that contain single and two-strain samples. The first subset contains eight single strain and eight two-strain samples with proportions varying from $50 \%$ to $95 \%$ in $5 \%$ increments, with $55 \%$ and $60 \%$ omitted. All the samples in the first subset have ten SNPs relative to H37Rv, and since these SNPs are chosen independently at random, the two-strain samples are 20 SNPs apart. The remaining sample subsets have the same proportions, but more SNPs. The genetic distances between mixed strains in each of the subsets are thus 20, 30, 40 and 50 SNPs, respectively. The WGS data is produced by the ART simulator with the same settings used to generate Dataset C except for the depth of coverage, which is set to 60 for all the samples.

\section{Required input data}

The Splitstrains pipeline uses the BWA-MEM tool, which makes use of paired-end information to produce the alignment. The current pipeline only keeps those pairs for which both elements have been mapped, in order to reduce the possibility of errors. Hence, Splitstrains only requires a BAM file of the paired-end data, a reference genome, and optionally, a generic feature format (GFF) file. We say that a sample represented in the BAM file contains a single strain if it is pure, a pair of strains called major strain and minor strain if it is a mixture of two strains, or multiple strains in the case of a mixture of more than two strains.

\section{Data pre-proccessing}

All datasets are pre-processed with Trimmomatic [18]. The Trimmomatic settings are:

ILLUMINACLIP=TruSeq3-PE-2,

SLIDINGWINDOW $=4$ with trimming threshold $=16$,

Leading $=10$,

Trailing=10,

Minlen=40.

\section{Feature vector construction}

The BAM file contains the alignment information for each individual read of a sequenced organism. We first convert the BAM file into a pileup format using the pysam library [19]. This pileup format summarises the alignment information for each individual base of the reference genome. We then construct a per-base feature vector defined as follows:

$$
\boldsymbol{x}_{i}:=\left(p_{A}^{(i)}, p_{C}^{(i)}, p_{G}^{(i)}, p_{T}^{(i)} ; d^{(i)}\right),
$$

where $p_{b}^{(i)} \in[0,100]$ for $b \in\{A, C, G, T\}$ is the percentage of base $b$ at position $i$, so that $\sum_{b \in\{A, C, G, T\}} p_{b}^{(i)}=100$, and $d^{(i)}$ is the total depth (number of aligned reads) at this position. Note that if $p_{b}^{(i)}>0$ for more than one $b$, there could be a SNP at position $i$. In practice, at most two of the $p_{b}^{(i)}$ 's are non-zero most of the time. In the absence of sequencing errors, we expect exactly one of the $p_{b}^{(i)}$ s s to equal 100 for every in the case of a single strain. On the other hand, in the case of a mixed sample that has a major strain at a proportion $p \geq \frac{1}{2}$ and a minor strain at a proportion $1-p$, we expect to see $p_{b_{i}}^{(i)}=p$ and $p_{b_{i}^{\prime}}^{(i)}=1-p$, where $b_{i} \neq b_{i}^{\prime}$, for sufficiently many positions $i$.

Fig. 1 shows an example with two adjacent positions, $i$ and $j$. There are $n=8$ reads supporting position $i$, six of them containing an $A$ and the remaining two containing a $T$. The depth is $d^{(i)}=8$ and the percentages of bases $\mathrm{A}$ and $\mathrm{T}$ at are $p_{A}^{(i)}=75$ and $p_{T}^{(i)}=25$, respectively. The adjacent position 


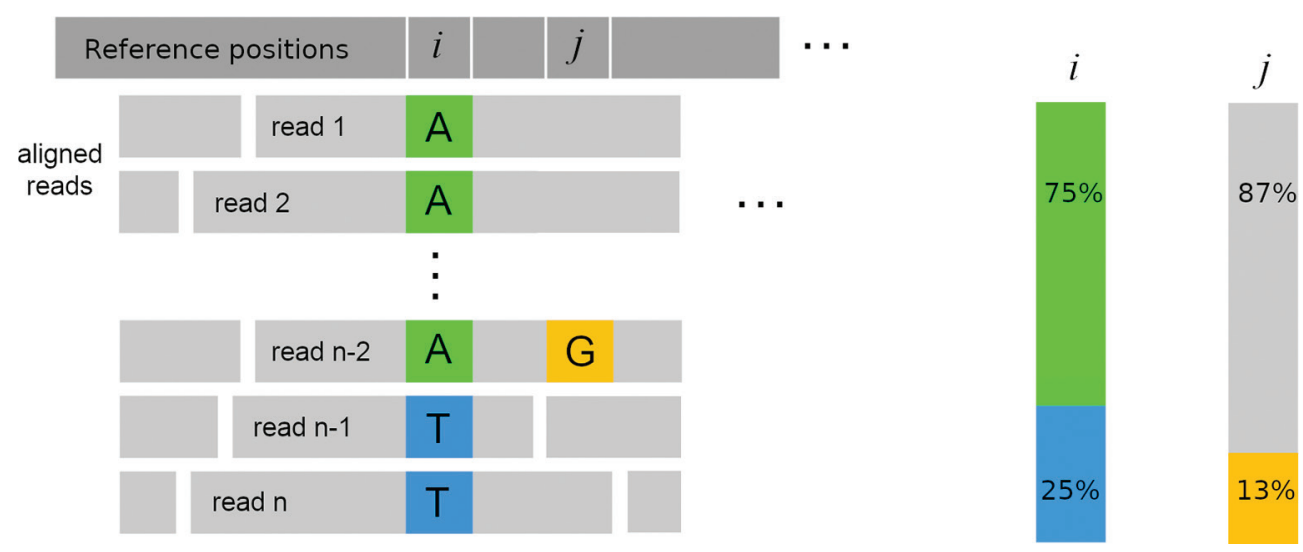

Fig. 1. An example read alignment and the corresponding feature vector.

$j$ has the same depth and a $G$ in one of the reads, with the remaining reads containing a $C$. They are summarized by the feature vectors $\boldsymbol{x}_{\boldsymbol{i}}=(75,0,0,25 ; 8)$ and $\boldsymbol{x}_{\boldsymbol{j}}=(0,87.5,12.5,0 ; 8)$, respectively.

We empirically observed that sites with a relatively low depth of coverage contained reads that could not be reliably aligned by BWA-MEM (i.e. they had poor alignment quality scores). For this reason, we chose to filter out any site $\boldsymbol{x}_{i}$ with depth coverage $d^{(i)}$ below $k=70 \%$ of the mean depth of coverage. Additionally, we use a GFF file based on the $M$. tuberculosis reference genome (NC 000962.3), but with the mobile and PE/PPE genes removed. This user-customizable GFF file ensures that Spl it Strains analyses only annotated gene regions excluding mobile and PE/ PPE genes, as the latter are known to be highly repetitive and produce unreliable alignment and variant calling results [20].

\section{Detecting mixed samples}

We test two hypotheses: the null hypothesis $\left(H_{0}\right)$, which states that there is a single strain in the data, and an alternative hypothesis $\left(H_{1}\right)$, which states that there are two strains with proportions $p$ and $1-p$, respectively. Our data $D$ consists of all the feature vectors $\boldsymbol{x}_{i}$ described above.

The likelihood of the data $D$ under $H_{0}$ is based on the fact that, for every position $i$, the feature vector $\boldsymbol{x}_{i}$ can only have a non-zero percentage at one base $b$, that is $p_{b}^{(i)}=100$. That is, under the null hypothesis, the maximum likelihood estimator of the underlying base given a single strain would always be the most frequently observed nucleotide. However, we also allow sequencing errors to occur with probability $\epsilon_{0}$. Therefore, using the notation $d_{i}$ for the number of reads that map to position $i$, and $k_{i}$ for the number of those reads that end up with the most frequent base, we have

$$
P\left(D \mid H_{0}\right)=\prod_{i}\left(\begin{array}{l}
d_{i} \\
k_{i}
\end{array}\right) \epsilon_{0}^{d_{i}-k_{i}}\left(1-3 \epsilon_{0}\right)^{k_{i}} \text {, with } k_{i}:=d_{i} \cdot \max _{b} p_{b}^{(i)} . \text { (2) }
$$

where the last term in the product arises from the fact that a sequencing error can occur in three different ways, so the probability of getting the correct base is $1-3 \epsilon_{0}$.

Under $H_{1}$ we assume that there are two strains with proportions $p$ and $1-p$, and sequencing errors which occur with probability $\epsilon_{1}$. We now use $n_{M}^{(i)}:=d_{i} \cdot \max _{b} p_{b}^{(i)}$ and $n_{m}^{(i)}:=d_{i} \cdot \max _{b^{\prime} \neq b} p_{b}^{(i)}$ to denote the counts of the most and second most frequent bases at position $i$, and $n_{e}^{(i)}:=d_{i}-\left(n_{M}^{(i)}+n_{m}^{(i)}\right)$ to denote the count of the remaining bases.

Repeating the above analysis gives us the following expression:

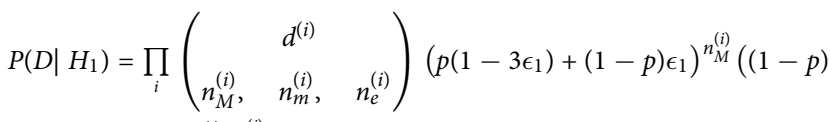

$$
\begin{aligned}
& \left.\left(1-3 \epsilon_{1}\right)+p \epsilon_{1}\right)^{n_{m}^{(i)}} \epsilon_{1}^{n_{e}^{(i)}},
\end{aligned}
$$

where

$$
\left(\begin{array}{ccc} 
& d^{(i)} & \\
n_{M}^{(i)}, & n_{m}^{(i)}, & n_{e}^{(i)}
\end{array}\right):=\left(\begin{array}{c}
d^{(i)} \\
n_{M}^{(i)}
\end{array}\right)\left(\begin{array}{c}
d^{(i)}-n_{M}^{(i)} \\
n_{m}^{(i)}
\end{array}\right)
$$

is the trinomial coefficient, which generalizes the binomial coefficient.

Using the likelihood ratio test, we can now formulate the following condition:

if $-2\left(\log \left(P\left(D \mid H_{0}\right)\right)-\log \left(P\left(D \mid H_{1}\right)\right)\right)<c$, then select $H_{0}$, otherwise $H_{1}$.

The threshold value $c$ is defined based on the significance level $\alpha$ using the $\chi^{2}$ distribution with one degree of freedom. Finally, in order to evaluate Equation (5) we estimate the parameters $\epsilon_{0}$ for $H_{0}$ and $p, \epsilon_{1}$ for $H_{1}$ using the well-established Truncated Newton constrained optimization algorithm [21]. In both cases we estimate a set of parameters that maximize the likelihood of the data conditional on the hypothesis, i.e. 
we perform maximum likelihood estimation on the corresponding parameters.

\section{Read assignment with two strains}

In order to obtain a read assignment to a strain, we wish to compute the probability of a read $r$ belonging to the major strain $M$ and to the minor strain $m$. Let $\operatorname{Pr}[r \in M]$ and $\operatorname{Pr}[r \in M]$ denote the respective probabilities. A read often supports multiple SNPs at the same time, say, at positions $i_{1}, i_{2}, \ldots, i_{n}$; we let $C_{r}=\left\{i_{1}, i_{2}, \ldots, i_{n}\right\}$ be the set of such positions for a read $r$.

For each $i \in C_{r}$ we get the counts of each base $b \in\{A, C, G, T\}$ from the feature vector $\boldsymbol{x}_{\boldsymbol{i}}$. Let $x^{(i)}$ denote the count of the base $r_{i}$ found in the read $r$ at position $i \in C_{r}$. We then define the following condition:

$$
\text { if } \frac{\operatorname{Pr}\left[r \in M \mid x^{(i)}, i \in C_{r}\right]}{\operatorname{Pr}\left[r \in m \mid x^{(i)}, i \in C_{r}\right]} \geq 1 \text {, then } r \in M \text {, otherwise } r \in m \text {. }
$$

We now analyse Equation (6) under two alternative models - a binomial one and a Gaussian one with equal variances (which would be the case if the Gaussian model was approximating the binomial one because $\sigma_{1}^{2}=d^{(i)} p(1-p)=d^{(i)}(1-p) p=\sigma_{2}^{2}$. We show that both of these models simplify to a majority vote of the variants present inside a read, where the $i-$ th variant's number of votes for a strain equals to its frequency in the feature vector, $\boldsymbol{x}_{\boldsymbol{i}}$. However, these votes are unweighted in the binomial model, while in the Gaussian model, each variant's total number of votes is normalized to one by dividing it by the depth of coverage $d^{(i)}$.

Suppose that the proportions of the major and the minor strains are $p$ and $1-p$, respectively, as inferred from the mixture model. Since $p$ is the proportion of the major strain, we may assume that $p \geq \frac{1}{2}$. To further simplify the computation, we assume that each $\boldsymbol{x}_{\boldsymbol{i}}$ is independent. Then the left-hand side of Equation (6) can be expressed as a ratio of products:

$$
\text { if } \frac{\prod_{i \in C_{r}}\left(\begin{array}{l}
d^{(i)} \\
x^{(i)}
\end{array}\right) p^{x^{(i)}}(1-p)^{d^{(i)}-x^{(i)}}}{\prod_{i \in C_{r}}\left(\begin{array}{l}
d^{(i)} \\
x^{(i)}
\end{array}\right)(1-p)^{x^{(i)}} p^{d^{(i)}-x^{(i)}}} \geq 1 \text {, then } r \in M \text {, otherwise } r \in m \text {. }
$$

Simplifying the left-hand side further, we arrive at a simple condition, independent of $p$ :

$$
\text { if } \sum_{i \in C_{r}}\left(2 x^{(i)}-d^{(i)}\right) \geq 0 \text {, then } r \in M \text {, otherwise } r \in m \text {. }
$$

Therefore, by applying Equation (8) we can classify whether a read $r$ belongs to the major or the minor strain. In the case of a perfect tie, we assign the read to the major strain.

If, instead of the derivation above, we use the Gaussian probability density functions $f\left(x \mid \mu, \sigma_{1}\right)$ and $f\left(x \mid 1-\mu, \sigma_{2}\right)$ to model the ratios in Equation (6), and further assuming equal variances $\sigma=\sigma_{1}=\sigma_{2}$, we arrive at a condition very similar to that in Equation (8):

$$
\frac{\prod_{i \in C_{r}} f\left(x^{(i)} / d^{(i)} \mid \mu, \sigma\right)}{\prod_{i \in C_{r}} f\left(x^{(i)} / d^{(i)} \mid 1-\mu, \sigma\right)} \geq 1 \text { iff } \sum_{i \in C_{r}}\left(2 x^{(i)} / d^{(i)}-1\right) \geq 0 .
$$

Although similar, Equations (8) and (9) can lead to opposite conclusions when the depth of coverage $d^{(i)}$ varies between the positions occurring in a read; for instance, when a read $r$ contains two biallelic positions, with the first one occurring in six reads of which five (including $r$ ) agree with the major strain, and with the second one occurring in nine reads of which two (including $r$ ) agree with it, then the binomial model would assign this read to the minor strain (since $2(5+2)=14<15=6+9)$, while the Gaussian model would assign it to the major strain (since 5/6+2/9 $=19 / 18>1)$. On the other hand, it is easy to see that if the coverage is uniform, i.e. $d^{(i)}=D$ for every position in the read, then Equation (8) and Equation (9) are equivalent. Hence, it is possible to use either the binomial or the Gaussian distribution in Equation (6), with identical assignments when we assume uniform coverage and equal variances and normalize the means to add up to one. In our implementation we choose not to constrain the variances to be equal, and apply the left-hand side of Equation (9) with the empirically fitted mean and variance, but with the fitted means normalized by their sum prior to the computation. This leads us to the more general situation, where we have $n>2$ strains in a sample.

\section{Binomial and Gaussian mixture models for multiple strains}

In this subsection we describe a probabilistic model for proportion estimation and read assignment in the case of multiple strains.

In order to build the Mixture Model, we use the information in the feature vectors $\boldsymbol{x}_{i}$ to construct a matrix $X$ as follows

$$
X:=\left[p_{A}^{\left(i_{1}\right)}, p_{C}^{\left(i_{1}\right)}, p_{G}^{\left(i_{1}\right)}, p_{T}^{\left(i_{1}\right)}, \ldots, p_{A}^{\left(i_{N}\right)}, p_{C}^{\left(i_{N}\right)}, p_{G}^{\left(i_{N}\right)}, p_{T}^{\left(i_{N}\right)}\right],
$$

where $\left\{i_{n}\right\}_{n=1}^{N}$ is a strictly increasing sequence of integer indices (i.e. $i_{1}<i_{2}<\ldots<i_{N}$ ) of variable positions. Now, let $K$ be the number of strains in a sample. We denote the unknown proportions of each strain as $\mu_{1}, \ldots, \mu_{K}$, the standard deviation of each proportion as $\sigma_{1}, \ldots, \sigma_{K}$. and the weight of each mixture model component, as $w_{1}, \ldots, w_{K}$. Define the parameter vector $\boldsymbol{\theta}:=\left\{\mu_{1}, \sigma_{1}, w_{1}, \ldots, \mu_{K}, \sigma_{K}, w_{K}\right\}$; then the mixture model has the following form:

$$
p(\boldsymbol{\theta} \mid X)=\sum_{k=1}^{K} w_{k} f\left(x \mid \mu_{k}, \sigma_{k}\right),
$$

where $f\left(x \mid \mu_{k}, \sigma_{k}\right)$ is the density function of a binomial or Gaussian distribution.

For a given $X$, we use the well-established EM (expectation maximization) algorithm to learn $\boldsymbol{\theta}$.

Once the model has been learned (Fig. 2), it is possible to proceed to the assignment of each read to a strain via Naïve Bayes classification:

$$
\mathbf{p}_{k}=\operatorname{Pr}\left[r \in S_{k} \mid p_{r_{i}}^{(i)} \text { for } i \in C_{r}\right] \propto w_{k} \prod_{i \in C_{r}} f\left(p_{r_{i}}^{(i)} \mid \mu_{k}, \sigma_{k}\right) .
$$




\section{Dataset $\mathrm{X}$ histogram}

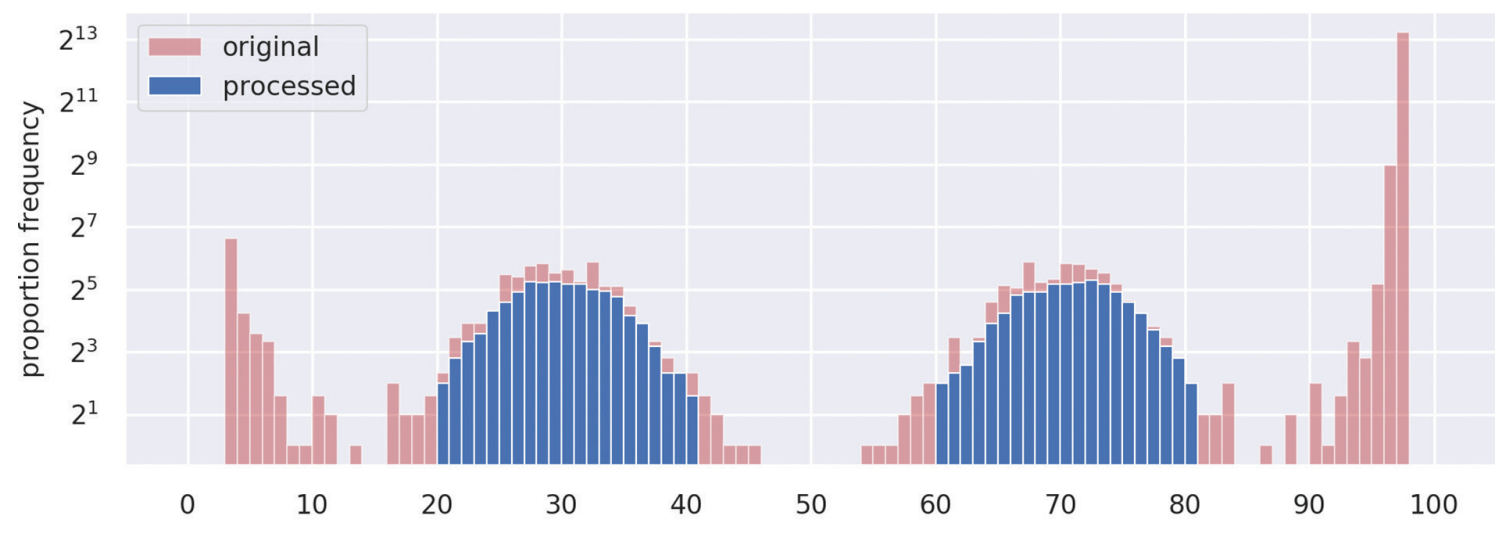

\section{Mixture Model}

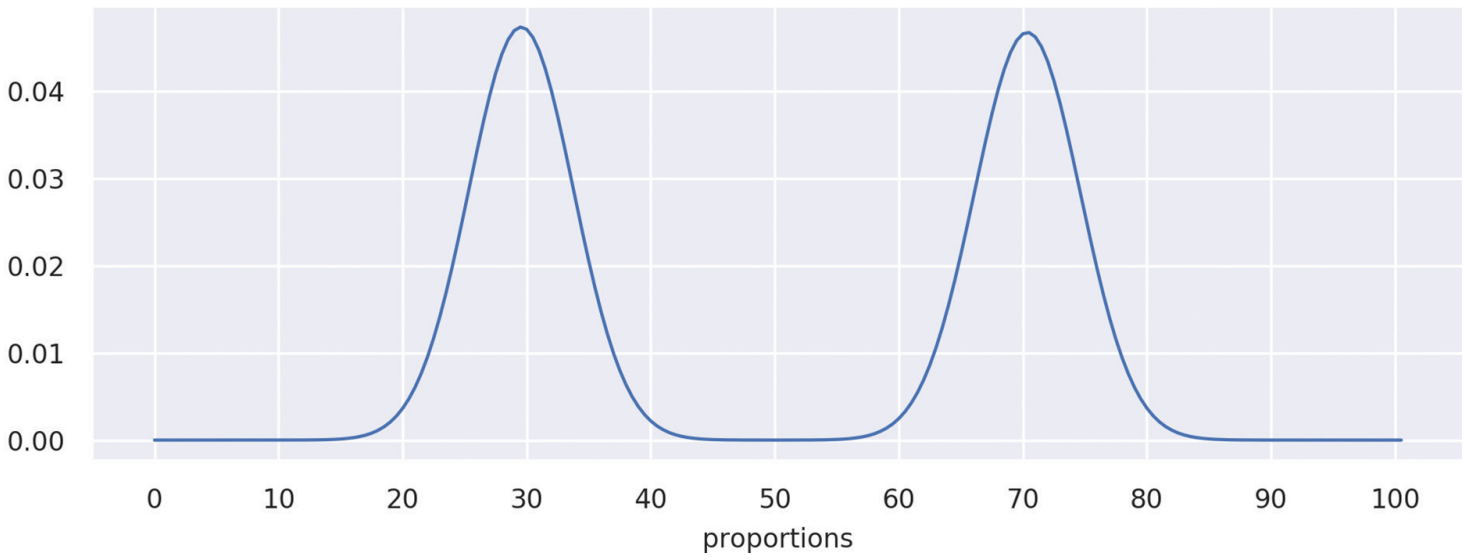

Fig. 2. Example of a simulated mixed sample, the data $X$, containing a major and a minor strain with respective percentages $\mu_{1}=70, \mu_{2}=30$. The red bars represent filtered out sites $\boldsymbol{x}_{\boldsymbol{i}}$ with depth $d^{(i)}$ less than $\kappa=70 \%$ of the average depth of coverage.

Finally, we use a maximum a posteriori assignment: $r \in S_{j}$ if and only if $\boldsymbol{p}_{j}=\max \left\{\boldsymbol{p}_{k}\right\}$.

\section{Computational settings}

All our computations are performed on a 64-bit Ubuntu Linux computer with eight CPU cores and 16 GB of RAM. The entire pipeline's running time ranges from 2 minutes to 30 minutes per sample, depending on the settings and the depth of coverage.

\section{RESULTS}

For simplicity, a WGS sample will be called pure if it contains a single strain of the sequenced organism and mixed otherwise. The Splitstrains algorithm classifies a sample as being pure or mixed. If the sample is classified as mixed, the algorithm detects the proportion of each strain and separates the reads according to which strain they belong to. In order to accomplish this, the algorithm proceeds through three stages.

First, SplitStrains uses the sample's SNPs to infer the parameters of a Gaussian or binomial Mixture Model (GMM), which identifies the number and the proportions of the constituent simple strains. The likelihood ratio statistic produced in the process provides a rigorous quantification of the confidence about its status as a pure or mixed sample. The algorithm then uses the model's estimated parameters in a Naive Bayes classifier to assign each read that contains variable positions to one strain. Finally, it produces a BAM file for each constituent strain. Note that any read that does contain any variable positions does not get classified, and instead gets included in all the BAM files produced. The process is shown in Fig. 3.

In principle, it would be possible for SplitStrains to partition the SNPs instead of the reads. However, we choose 


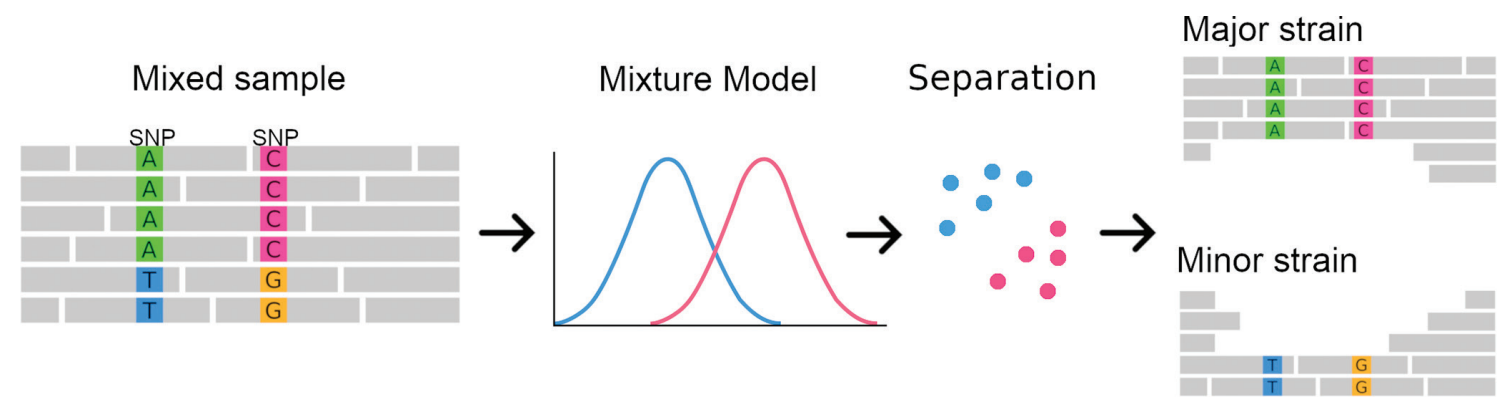

Fig. 3. Splitstrains workflow overview.

to partition the reads for two reasons. First, there is generally more information to classify a read than there is to classify a SNP, because a single read can contain multiple SNPs, as we explain in the Methods. Second, an assembly program (either reference-based or de novo) can be applied directly to the BAM files representing each strain, which can make the downstream analysis more accurate in cases where the variation present in some of the strains is not limited to SNPs, but also includes indels or copy number variants.

Although Splitstrains is primarily designed for samples containing one or two strains, we also apply it to situations, rarely seen with $M$. tuberculosis but more common with other bacteria, where three strains are present in a single sample. We note that our likelihood ratio statistic is calibrated to distinguish between the pure and the mixed case but is not in general sensitive enough to distinguish between two-strain and three-strain samples. For this reason, we consider our mixed sample detection to be correct if it classifies a mixed sample as mixed, regardless of the number of underlying strains.

To evaluate the strain proportion estimation in a uniform way, we consider the accuracy of the proportion estimation for the major strain (which, in the case of a two-strain sample, is equivalent to the accuracy for the minor strain). Lastly, to evaluate the assignment of reads to constituent strains, we separately analyse the samples with two and three strains, and explicitly specify the number of constituent strains as two or three.

We evaluate the performance of the Splitstrains algorithm by quantifying the accuracy of its mixture proportion estimation and strain separation on several datasets:

- Dataset A 48 in vitro samples, including 12 pure and 36 artificially mixed two-strain samples with known strain proportions, published together with a previous method, MixInfect [10]. The samples have an average of 1249 SNPs relative to the reference genome. This dataset is the most realistic representation of mixed infections since it is generated in vitro by combining the DNA from two $M$. tuberculosis cultures.

- Dataset B 60 in silico samples, including 30 pure and 30 mixed two-strain samples with known strain proportions, published together with a study on whole-genome MLST schemes from our group [14]. The samples have an average of 6375 SNPs relative to the reference genome. This dataset is the second most realistic dataset since it is based on previously observed alleles of genes and includes a wide range of different mixture proportions.

- Dataset C 22 in silico samples, of which eight are pure and 14 are mixed (eight with two and six with three constituent strains) produced specifically for this work. The mixed samples are derived directly from the reference genome by independently adding 100 SNPs per strain in the twostrain samples, and 300 SNPs per strain in a three-strain sample. This is the least realistic dataset, and it is designed to test Splitstrains's ability to generalize to data with one, two or three strains and a wide range of mixture proportions.

- Dataset D 59 real samples chosen among those collected in population-level surveys in Azerbaijan, Bangladesh, Belarus, Pakistan, Philippines, South Africa (Gauteng and Kwazulu Natal provinces) and Ukraine, collected between 2009 and 2014 [22], with additional samples from a large-scale whole genome sequencing study conducted in Malawi [23]. The samples have an average of 1248 SNPs relative to the reference genome. This dataset contains real samples, and their true label (pure or mixed) is unknown.

- Dataset E 64 in silico samples, 32 pure ones and 32 mixed ones with two strains each, used to test the method at a low depth of coverage (60) and a low genetic distance. The 32 mixed samples have one of eight known major strain proportions, and for each proportion, the samples are derived from the reference genome by independently adding 10 , 15,20 , or 25 SNPs per strain. This is a calibration dataset designed to test SplitStrains's ability to detect mixed infections with very short genetic distances.

The analysis starts by classifying a given sample as mixed or pure. It uses the likelihood ratio test (LR) to compare the single strain (null) and the multiple strains (alternative) hypotheses. We use the LR statistic to guide the decision process. The algorithm also reports the estimated mixture proportions. If the sample is called mixed, the algorithm further classifies each read containing one or more variants as belonging to a specific strain. Note that this assignment can 
be extended to a full strain assembly as is frequently done in metagenomics [24], but we do not explicitly do so to focus on our contribution and avoid the complications due to the choice of a suitable assembly algorithm [25]. We now discuss the performance of SplitStrains on each of the datasets, omitting the real Dataset $\mathrm{D}$ which cannot be used for evaluation due to the absence of "gold standard" information.

\section{Mixed sample detection}

SplitStrains is consistently able to detect mixed infections across all the datasets, which suggests its robustness to different numbers of SNPs, depths of coverage, minor strain proportions, and synthesis methods.

Dataset A SplitStrains correctly classifies all 12 pure samples when the significance level threshold set to $\alpha=0.05$. Then 23/24 mixed samples with major strain proportions of 70 and $90 \%$ are also correctly classified. 11/12 samples with a major strain proportion of $95 \%$ are misclassified as pure; however, by increasing the significance level threshold to $\alpha=0.1$, SplitStrains correctly classifies $8 / 12$ of these samples, while the classification results for the 24 mixed samples remain unchanged. In total, $43 / 48$ samples (90\%) get correctly classified with $\alpha=0.1$.

Dataset B SplitStrains classifies 29/30 mixed samples as mixed, the exception being a sample with a major strain proportion of $95 \%$. However, using $\alpha=0.1$ instead of $\alpha=0.05$ allows for the correct classification of all 30 mixed samples. Then $27 / 30$ pure samples get classified as pure with $\alpha=0.1$, and the three misclassified samples have a very low average depth of coverage (10). In total, 57/60 samples (95\%) get correctly classified with $\alpha=0.1$.

Dataset C SplitStrains correctly detects $13 / 14$ mixed samples using $\alpha=0.05$, including $6 / 6$ samples with three constituent strains. The misclassified sample is the two-strain sample with a major proportion of $95 \%$. All 8 pure samples are correctly classified as pure. In total, 21/22 samples (95\%) get correctly classified with $\alpha=0.05$.

Dataset D We also applied Splitstrains to a real dataset containing 59 samples. A preliminary analysis used a reference mapping pipeline with variant calling optimized on curated databases [26], and declared 27 of the samples as mixed. Splitstrains classifies $24 / 27$ of these samples as mixed with $\alpha=0.05$, and classifies the remaining 35 samples as pure, demonstrating a concordance of $56 / 59$ (95\%) with the reference mapping pipeline that makes extensive use of database information.

\section{Mixture proportion estimation}

In those datasets where the true proportion of the major strain is known, we can compare that proportion to the one inferred by Splitstrains, conditional on its correctly classifying the sample as mixed. The correctly classified mixed samples have a maximum deviation from the true solution of $11 \%$; the worst case occurs for a sample with true major proportion of $95 \%$, which is estimated as $84 \%$ by Splitstrains. In general, the estimation is accurate up to a $90 \%$ major strain proportion but starts to decrease as this proportion

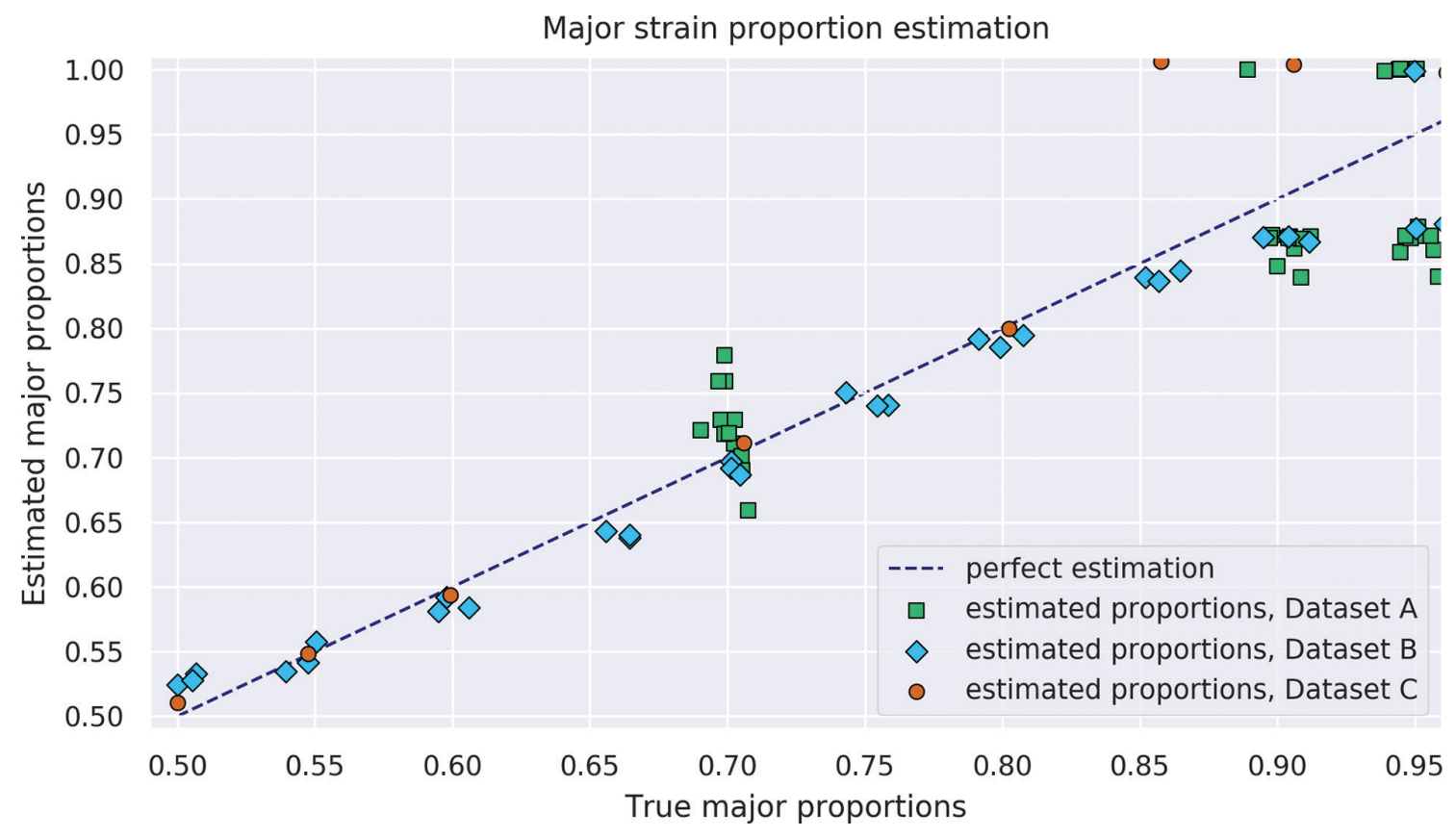

Fig. 4. Proportion estimation. The 74 true mixed samples and their major proportion estimated by SplitStrains. 
Table 1. RMSE comparison across all datasets

\begin{tabular}{|lccccc|}
\hline Dataset & Size (two-strains only) & SplitStrains & Mixed infection estimator & MixInfect & 0.178 \\
\hline A & 36 & $\mathbf{0 . 0 5 6}$ & 0.068 & 0.153 \\
B & 30 & 0.025 & $\mathbf{0 . 0 1 8}$ & 0.031 \\
C & 8 & 0.066 & 0.066 & 0.041 \\
Combined & 74 & $\mathbf{0 . 0 4 7}$ & 0.053 & 0.312 \\
\hline
\end{tabular}

approaches 95\% (Fig. 4). We measure the deviation of all the estimates from their true values using the Root Mean Square Error (RMSE). Averaged across all three datasets with known true proportions, the RMSE of Splitstrains is less than $5 \%$, as also shown in Table 1 below.

\section{Assignment of reads to constituent strains - Dataset C}

Once the mixture model parameters have been estimated, the algorithm assigns each read containing one or more variable sites to a constituent strain using a Naïve Bayes approach. Note that those reads that do not contain any variant sites or have zero map quality remain unassigned (i.e. we perform a partial, rather than complete, strain reconstruction). In Figs 5 and 6 we respectively present the two-strain and three-strain confusion matrices to show the performance of this assignment on Dataset $\mathrm{C}$, which is designed in such a way that the provenance of each read is known. We set $\alpha=0.05$ to resolve the mixed strains. As explained above, the two-strain mixed sample with $95 \%$ major strain proportion is misclassified as pure, so Fig. 5 only contains seven contingency matrices. Here, a read is deemed to be classified correctly when it is assigned to its own strain, and incorrectly otherwise. These figures suggest that our assignment accuracy decreases as the two major strain proportions get close to each other, for samples with both two as well as three strains.

In practice, even if major and minor strain proportions are well apart, say 70:30, each individual variant in a read alignment file rarely has a clean 70:30 allele split. Instead, a variant's allele proportions take on values which are approximately normally distributed with respective means 70 and 30 . Splitstrains successfully handles such variants. However, if a variant comprised of two alleles has an allele with a frequency below a user-specified threshold (the default being 10\%), such a variant is deemed to be noisy and is not processed.

Using the read assignments to the strains, the algorithm outputs a new alignment file for each strain. In order to further evaluate the accuracy of the assignment, we create a consensus sequence from each alignment file. We expect the consensus sequences to match the respective genomes of the constituent strains. As the genome of each constituent strain has the same number $N$ of base substitutions relative to the reference genome, due to the way they are generated, the consensus sequences can have between 0 and $N$ mismatches with the true sequences. In the case of the two-strain mixtures, our algorithm successfully separates the strains with major

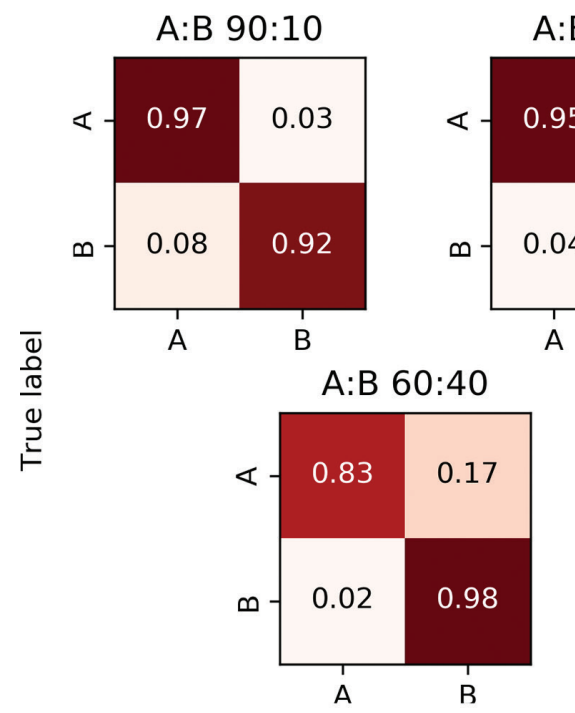

A:B 85:15
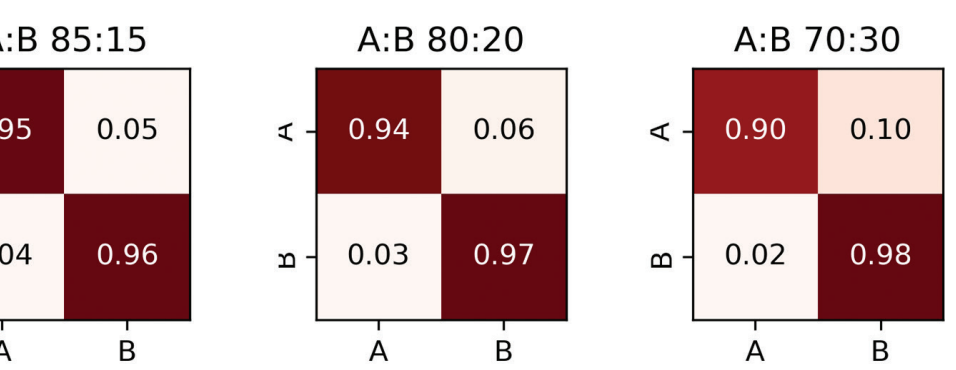

B
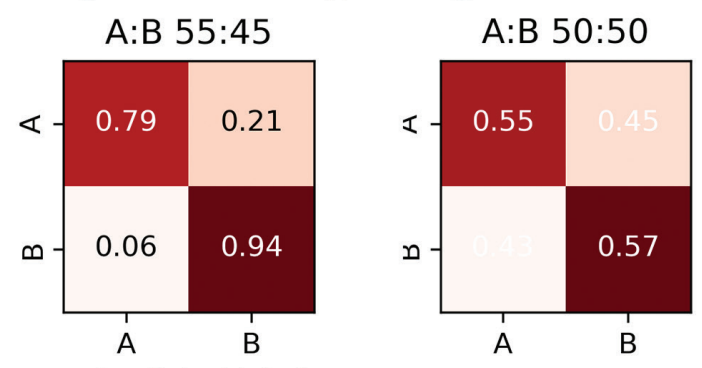

Predicted label

Fig. 5. Confusion matrices for two-strain samples, Dataset C. The major and minor strains are denoted A and B; their proportions are displayed above each matrix. 

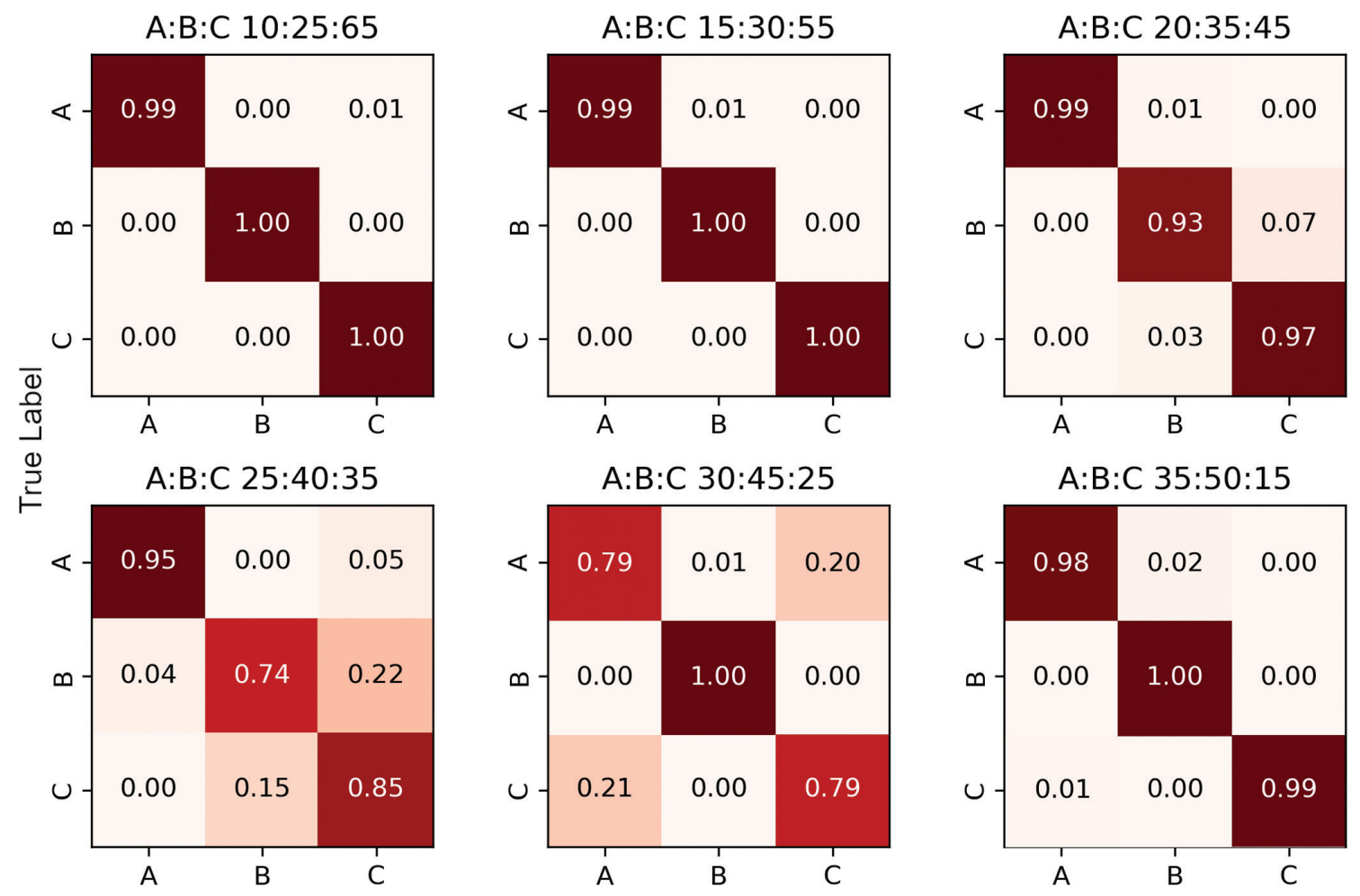

Predicted Label

Fig. 6. Confusion matrices for three-strain samples, Dataset C. The strains in each sample are denoted A, B and C; their proportions are displayed above each matrix.

strain proportion varying from $55 \%$ to $90 \%$. However, as the major strain proportion gets closer to $50 \%$, correct assignment of reads becomes steadily more challenging, as shown in Fig. 7. We note that SNPs in the reads that the alignment algorithm was unable to find a reasonable quality alignment for are not counted toward the errors in this analysis. Such reads are contained in the intervals [400130, 401700], [888990, 891520] and [2550020, 2551390] in the reference genome for M. tuberculosis, H37Rv (accession number NC 000962.3 at NCBI [12]), which fall within repetitive or mobile genome regions and are known to cause poor read alignments.

\section{Comparison with other tools}

SplitStrains consistently outperforms MixInfect [10], QuantTB [11] and Mixed Infection Estimator [2] in discriminating between pure and mixed infections. We illustrate their discrimination performance on datasets $\mathrm{A}, \mathrm{B}$ and $\mathrm{C}$ combined in Fig. 8; their performance on each of the datasets separately is shown in Fig. 9 in the Supporting Information. The Receiver Operating Characteristic (ROC) curve shows the true positive rate (TPR) against the false positive rate (FPR) at various threshold settings. The Area Under the Curve (AUC) quantifies how well the algorithm is able to distinguish between pure and mixed infections. Higher AUC values mean that the algorithm is better at predicting the class of a sample. The statistics used as inputs to the AUC computation are as follows: likelihood ratio for Splitstrains, proportion het/total for MixInfect, deviance for Mixed Infection Estimator, and internal totscore for Quant TB. Splitstrains has the highest AUC (0.99) and can achieve close to $100 \%$ TPR with an FPR as low as $11 \%$. The second best classification performance is obtained by MixInfect, with an AUC of 0.97. The ROC curves of Splitstrains and MixInfect are fairly close to one another, but the latter produces more false positives at higher true positive rates, giving a lower overall area under the curve.

Split Strains also consistently obtains the lowest or second lowest proportion estimation error among the tools, and has the lowest error on the combined dataset. These results are shown in detail in Fig. 10 and summarized in Table 1.

\section{Performance with low genetic distance and depth of coverage}

We test the performance of Splitstrains on Dataset E which contains 32 pure and 32 mixed strains with small genetic distances (20,30, 40 and 50 SNPs) and a low depth 


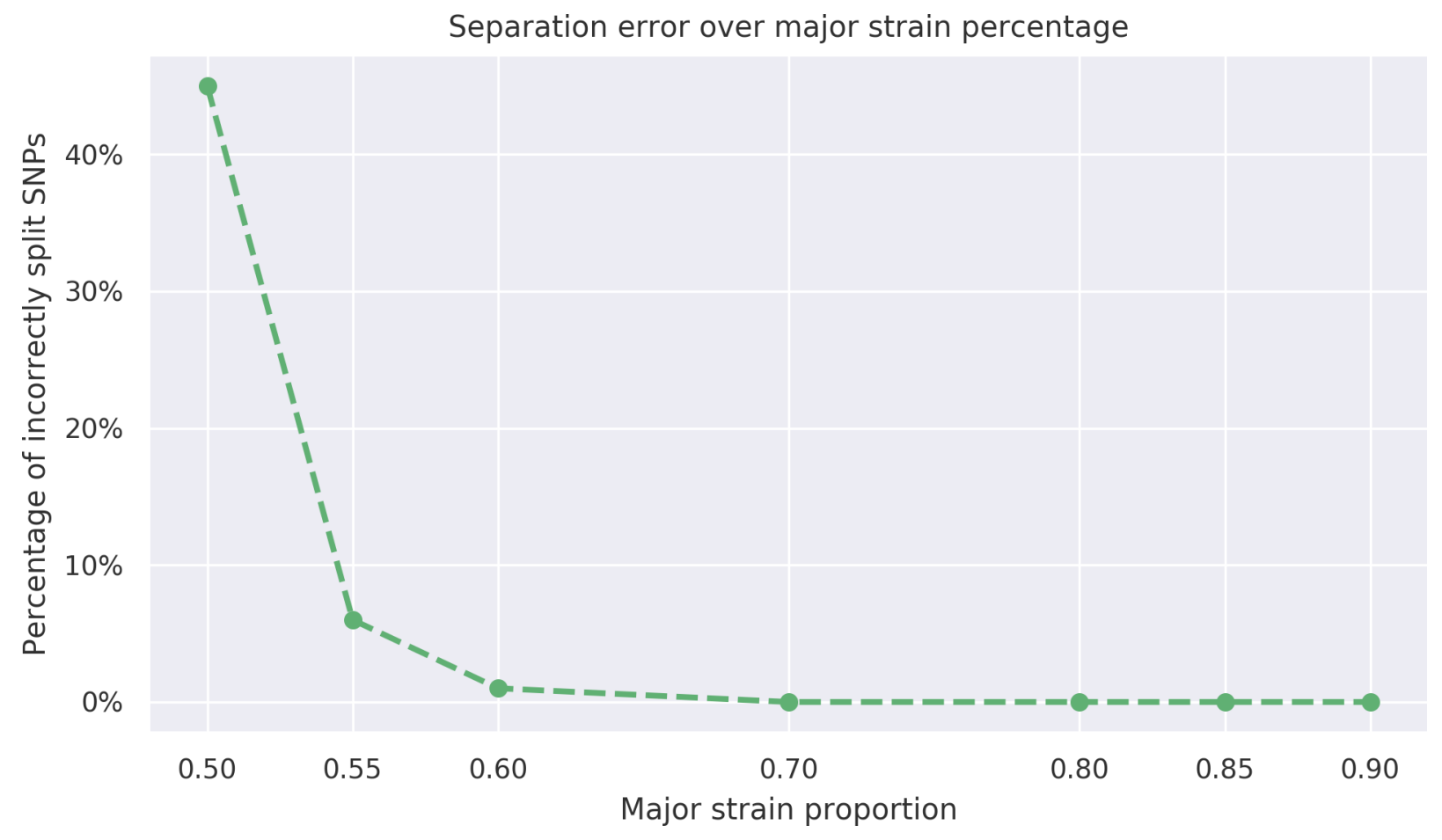

Fig. 7. Assignment error. The proportion of mismatches due to the incorrect assignment of reads among the positions where the strains differ from one another.

of coverage of 60. SplitStrains's ability to detect mixed infections in this setting depends on the threshold $\alpha$. Higher $\alpha$ values allow the detection of mixed strains with small genetic distances at the risk of classifying pure strains as mixed.
Fig. 11 shows the minimum $\alpha$ threshold needed to correctly identify mixed samples as mixed at a given SNP distance and major strain proportion while also correctly classifying

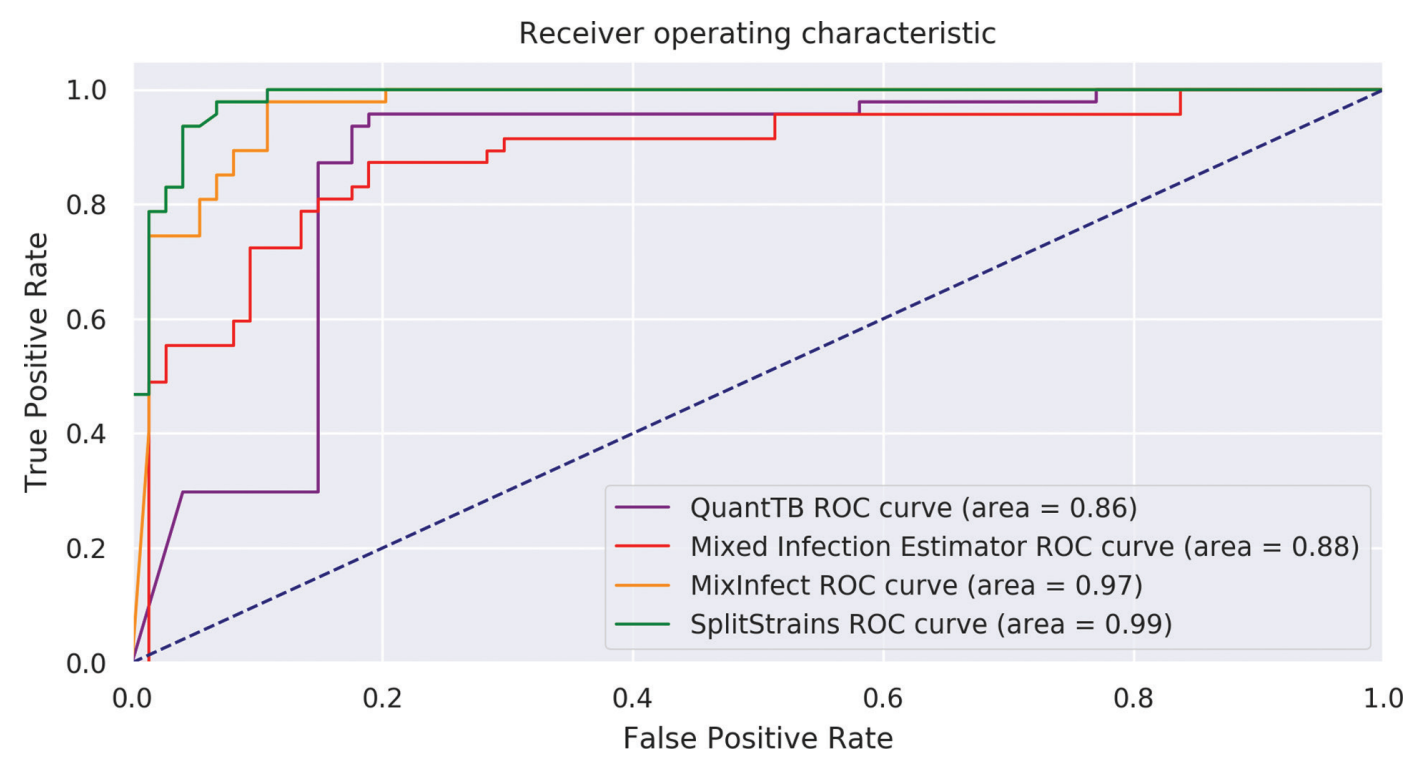

Fig. 8. ROC curves for the four tools. Performance in separating pure and mixed samples in datasets A, B and C. 


\section{Receiver Operating Characteristic}

Dataset A

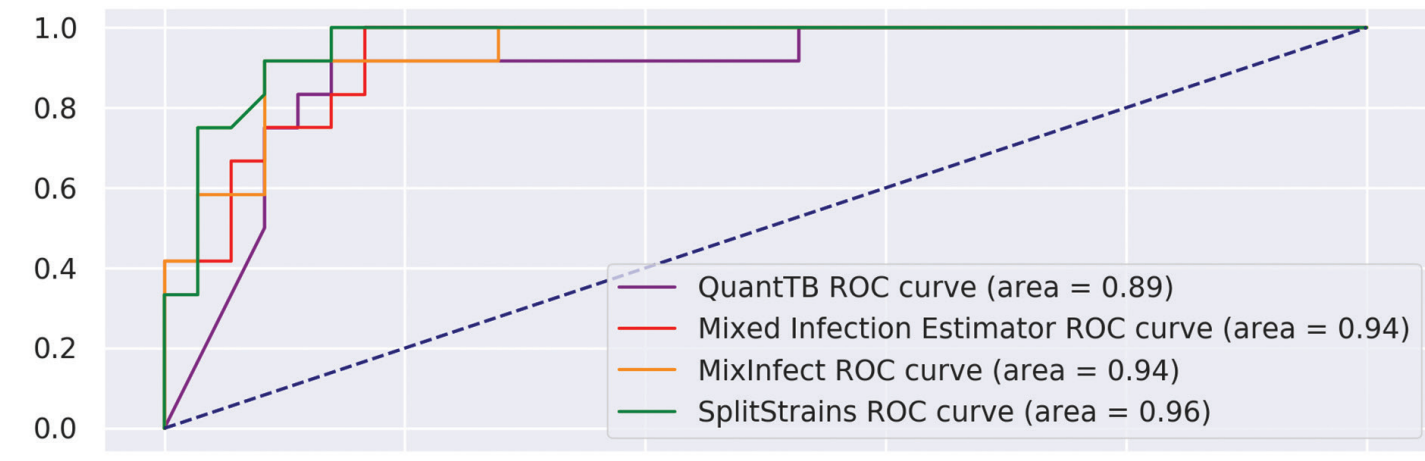

Dataset B

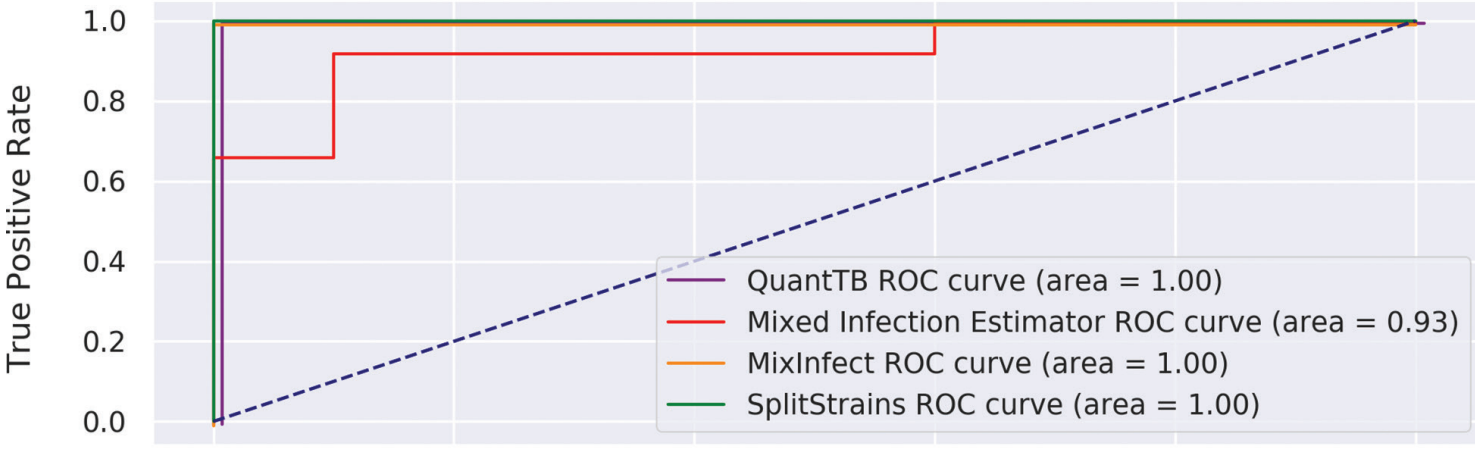

Dataset C

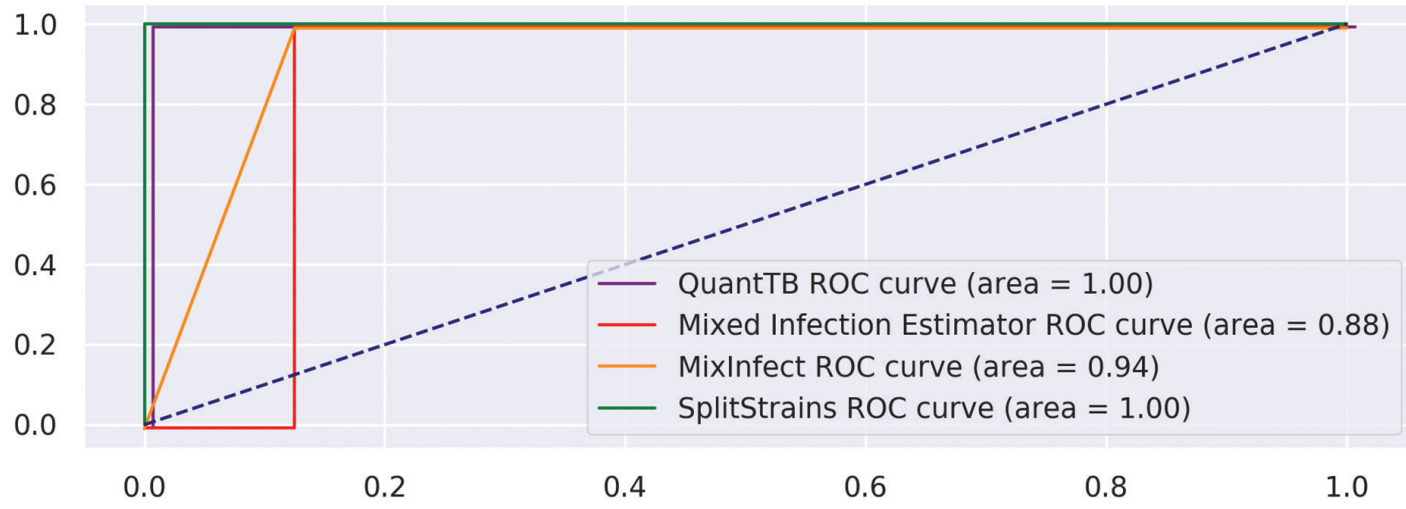

False Positive Rate

Fig. 9. ROC curves of all tools for each individual dataset A, B and C.

the pure samples as pure at the same SNP distance from the reference genome.

Fig. 11 shows that smaller genetic distances and larger major proportions require larger values of $\alpha$. For instance, a distance of 20 SNPs and a major strain proportion of $90 \%$ represents a challenging case which requires $\alpha$ to be set to 0.75 , while the default value, $\alpha=0.05$, suffices for a distance of 50 SNPs and a $50 \%$ major strain proportion.

We also show a separate ROC curve for Dataset E, in Fig. 12. Despite the challenges of classifying this Dataset, SplitStrains displays a reasonable trade-off between true positive and false positive rates and achieves an AUC of 0.96. 


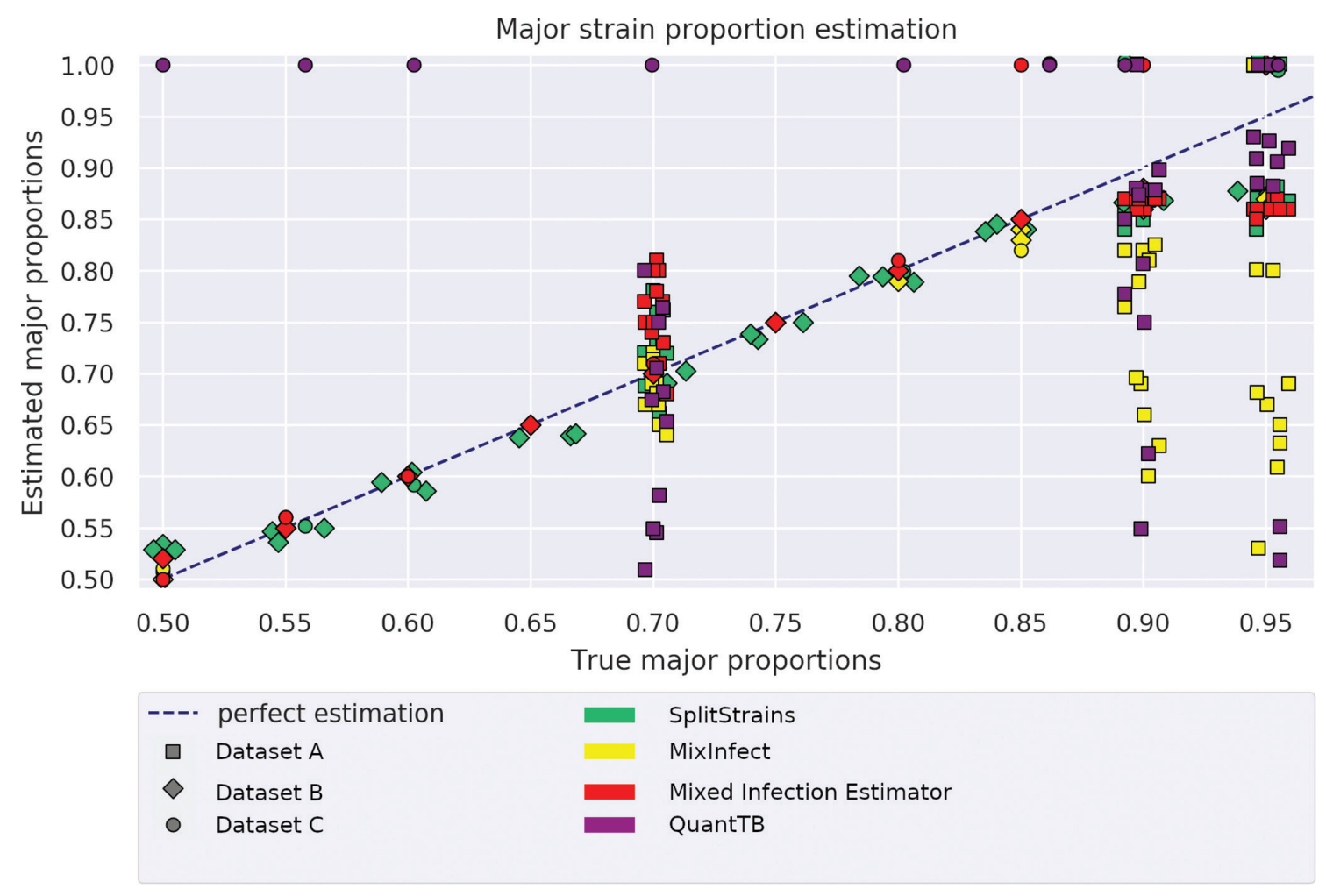

Fig. 10. Proportion estimation comparison. Major proportion estimates by four different tools on the 74 mixed samples from datasets $A, B$, and $C$.

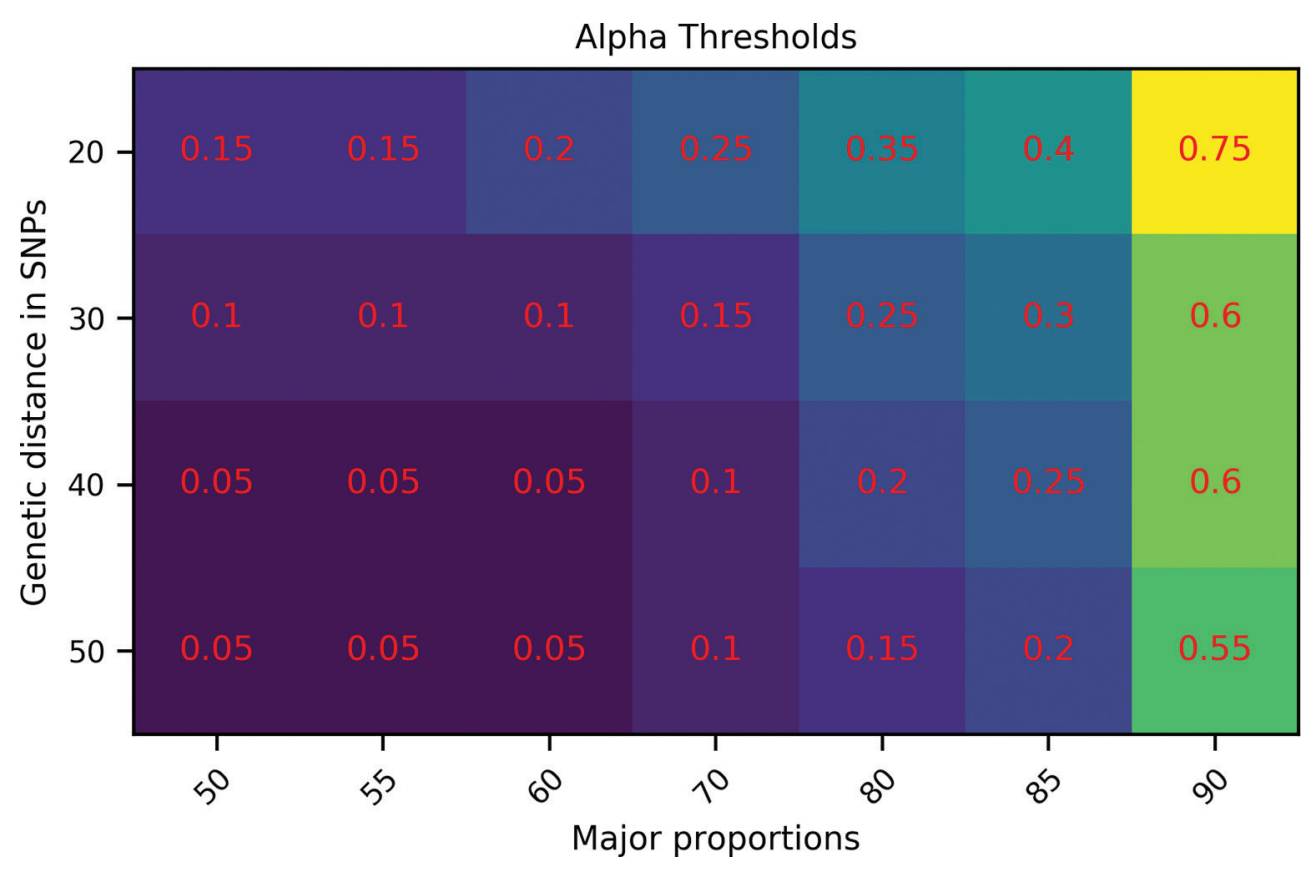

Fig. 11. $\alpha$ calibration, Dataset $E$. The $\alpha$ values required for the detection of mixed strains. 


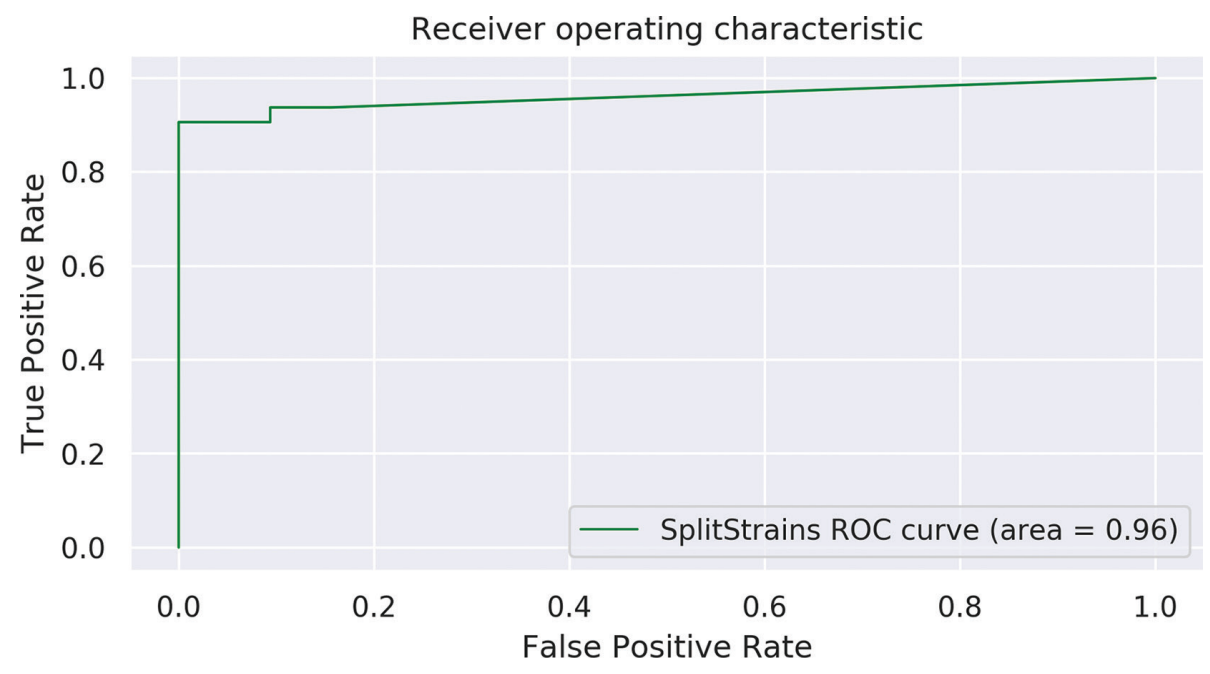

Fig. 12. ROC curve, Dataset E. The ROC curve for all 32 mixed and 32 pure samples.

\section{DISCUSSION}

In this paper we introduced a novel algorithm, called Splitstrains, based on a rigorous statistical framework, for detecting multiple-strain infections, estimating the proportion of the major and minor strains, and partially reconstructing their sequences by assigning the reads that contain variants to one of these strains.

The M.tuberculosis's genome is 4.4 million base pairs long and encodes roughly 4000 genes [27]. The genome features low amounts of recombination [28]. In addition, it has a high $\mathrm{G}+\mathrm{C}$ content, with nearly two-thirds of all the base pairs being G-C [27]. This makes Splitstrains not only applicable to $M$. tuberculosis, but to other clonal pathogens whose genomic diversity is low and in the absence of significant amounts of recombination. This is the case of genetically monomorphic pathogens like Yersinia pestis, Salmonella typhi, and Bacillus anthracis [29], and pathogens that exhibit clonal evolution during infections [30]. It is also possible that Splitstrains could be applied to more recombinogenic bacteria like Neisseria meningitidis [31], provided that the recombination hotspots are removed in a preprocessing step using a software such as ClonalFrameML [32].

Although successful in many simulation scenarios, our algorithm suffers from the following limitations:

- When the proportions of two of the strains are very close to one another (for instance, a 50-50 mixture of two strains), the estimation becomes numerically sensitive and the identified strain sequences cannot be reliably inferred.

- When the overall depth of coverage is too low (below 20 or so), the proportions and the strain sequences cannot be reliably estimated either.

- The multiple infection status cannot be determined with confidence when the proportion of one of the strains is below $10 \%$, which is therefore the resolution of our algorithm.

- Although there is no required minimum threshold on genetic distance between the strains in a mixed sample, those with a high genetic similarity (around 20 SNPs apart) are challenging to identify and deconvolute. This suggests that our method will likely perform better on mixed samples due to multiple infection events than those due to within-host evolution [1].

We now discuss these limitations in turn, and sketch possible ways to improve on them. The situation when two strains are present at equal or near-equal proportions is rare in practice, since the strains will in general have unequal fitness and one will tend to dominate the other [5]. However, if it does occur, Splitstrains provides a useful diagnostic in the proportions it returns, and this can then serve as a starting point for separately culturing and sequencing each of the strains.

The situation when the depth of coverage is too low also occurs infrequently in practice, since most modern sequencing experiments tend to have a depth of coverage of at least 75 to 100. In the case of a low depth of coverage, for instance in a historical sequencing experiment, it is frequently not possible to reliably determine the sequence of even a simple strain, due to the difficulty in differentiating between a true SNP and a sequencing error. As can be expected, this difficulty is exacerbated in the presence of multiple strains. A more time-consuming method, such as a de Bruijn graph-based assembly, may be able to address this limitation in some situations [33].

Similarly, a very low-frequency minor strain is typically difficult to distinguish from noise due to sequencing errors, and in a situation when this appears to happen, a more targeted approach such as amplicon sequencing may be used to establish the sequence of at least a subset of the important genes. This approach may be used, for instance, 
to determine the resistance to a specific drug of the major and the minor strain [34].

Lastly, mixed samples with a small SNP distance (around 20) between the constituent strains should arise primarily through within-host evolution, although there is also a small likelihood of reinfection by two very similar strains in a highprevalence region. Such similar strains may be more easily detectable by amplicon sequencing as well, provided that the sequencing is focused on the regions containing the variable positions. Alternatively, as we show in Fig. 11, the $\alpha$ significance level threshold in the Splitstrains algorithm can be increased to enable their detection in situations where such occurrences are expected to be frequent and the downside of false positive mixed calls is lower.

In spite of these limitations, we believe that our approach is a promising way forward, as also demonstrated by its favourable performance relative to existing methods. In particular, our approach appears to perform better than both Quant TB as well as Mixed Infection Estimator, and performs comparably to Mix In fect, in most simulated settings, with regards to the task of identifying the presence of mixed infections and estimating proportions.

In addition, SplitStrains is unique among existing methods in its ability to provide additional information, namely, the assignment of each read to one of the underlying strains, with a subsequent identification of their sequence if desired. Importantly, unlike QuantTB, it does not rely on the knowledge of a large number of previously identified sequences, which is a clear advantage when investigating either a novel outbreak or an isolate originating from a datapoor setting. Furthermore, Splitstrains returns not only a call, but also a likelihood ratio, which is an indicator of the algorithm's confidence about the presence or absence of a mixed infection. We believe that, in situations where such information has either clinical or public health importance, the SplitStrains method will be a valuable addition to the existing collection of tools.

In future work, we plan to extend SplitStrains to work with other bacterial pathogens as well as to improve its resolution, at least in datasets with a high depth of coverage. Lastly, we plan to use Splitstrains as a pre-processing step in two pipelines - one for identifying related isolates in an outbreak [35], where mixed infections can mask such relatedness, and another one for predicting drug resistance [36], where mixed infections can impede a correct prediction when only the minor strain is drug-resistant.

\section{Funding information}

This work has been funded in part by a CANSSI Collaborative Research Team grant, 'Statistical methods for challenging problems in public health microbiology' and a Genome Canada grant, 'Machine Learning Methods to Predict Drug Resistance in Pathogenic Bacteria'. LC acknowledges funding from a Sloan Foundation fellowship (FG-2016-6392) and the MRC Centre for Global Infectious Disease Analysis (reference MR/ R015600/1), jointly funded by the UK Medical Research Council (MRC) and the UK Foreign, Commonwealth and Development Office (FCDO), under the MRC/FCDO Concordat agreement, and is part of the EDCTP2 programme supported by the European Union. ML acknowledges funding from a NSERC Discovery grant.

\section{Acknowledgements}

The authors would like to acknowledge the invaluable input of Dr Cedric Chauve, Dr Theodore Cohen, Dr John Lees, Dr Nicholas Croucher, and Hooman Zabeti. They would also like to thank Dr Patrick Cudahy for beta-testing an earlier version of the tool.

\section{Author contributions}

E. G., methodology, software, validation, formal analysis, visualization, writing - original draft preparation, writing - review and editing. $M$. M. M., data curation, resources, writing - review and editing. I. C., data curation, resources, supervision, writing - review and editing. M. L., conceptualisation, methodology, formal analysis, supervision, funding, writing - review and editing. L. C., conceptualisation, methodology, formal analysis, supervision, project administration, writing - original draft preparation, writing - review and editing.

Conflicts of interest

The authors declare that there are no conflicts of interest.

\section{References}

1. Cohen T, Helden PD, Wilson D, Colijn C, McLaughlin MM, et al. Mixed-strain Mycobacterium tuberculosis infections and the implications for tuberculosis treatment and control. Clin Microbiol Rev 2012;25:708-719

2. Eyre DW, Cule ML, Griffiths D, Crook DW, Peto TEA, et al. Detection of mixed infection from bacterial whole genome sequence data allows assessment of its role in Clostridium difficile transmission. PLoS Comput Biol 2013;9:e1003059.

3. ShaJ, Almagro G, Mc V. Deconvolution of multiple infections in Plasmodium falciparum from high throughput sequencing data. Bioinformatics 2017;34:9-15.

4. Nathavitharana RR, Shi CX, Chindelevitch L, Calderon R, Zhang Z, etal.Polyclonal pulmonary Tuberculosisinfections and risk for multidrug resistance, LIMA, Peru. Emerg Infect Dis 2017;23:1887-1890.

5. Sergeev R, Colijn C, Cohen T. Models to understand the populationlevel impact of mixed strain M. Tuberculosis infections. J Theor Biol 2011;280:88-100

6. Weiss S, David S, Victor I. Heteroresistance: A cause of unexplained antibiotic treatment failure? PLOS Pathogens 2019;15:1-7.

7. Zong Z, Huo F, Shi J, Jing W, Ma Y, et al. Relapse versus reinfection of recurrent tuberculosis patients in a national Tuberculosis specialized hospital in Beijing, China. Front microbiol 2018:9:1858.

8. Nadon CA, Trees E, Ng L, Møller E, Reimer A, et al. Development and application of MLVA methods as a tool for inter-laboratory surveillance. Euro Surveill 2013;18.

9. Leonid C, Colijn C, Moodley P, Wilson D, Cohen T, et al. ClassTr: Classifying within-host heterogeneity based on tandem repeats with application to Mycobacterium tuberculosis infections. PLOS Computational Biology 2016:12:1-16.

10. Sobkowiak B, Glynn JR, Houben RMGJ, Mallard K, Phelan JE, et al. Identifying mixed Mycobacterium tuberculosis infections from whole genome sequence data. BMC Genomics 2018;19:613.

11. Anyansi C, Keo A, Walker BJ, Straub TJ, Manson AL, et al. QuantTB - a method to classify mixed Mycobacterium tuberculosis infections within whole genome sequencing data. BMC genomics 2020;21:80.

12. O'Leary NA, WrightM, Brister R, Ciufo S, Haddad D, et al. Reference sequence (Refseq) database at NCBI: Current status, taxonomic expansion, and functional annotation. Nucleic Acids Res 2016:44:D733-D745.

13. Moon TK. The expectation-maximization algorithm. IEEE Signal Process Mag 1996;13:47-60.

14. Feijao P, Yao H-T, Fornika D, Gardy J, Hsiao W, et al. MentaLiST - A fast MLST caller for large MLST schemes. Microb Genom 2018;4.

15. De J, Michael A, Gerrick E, Xu W, Park S, et al. Comprehensive essentiality analysis of the Mycobacterium tuberculosis genome via saturating transposon mutagenesis. MBio 2017;8:16-e02133. 
16. Huang W, Li L, Myers JR, Marth GT. Art: A next-generation sequencing read simulator. In: Bioinformatics, Vol. 28. Oxford University Press, 15 2012. 2012. pp. 593-594.

17. Li H. Aligning sequence reads, clone sequences and assembly contigs with BWA-MEM. arXiv 2013:1303.3997.

18. Bolger A, Lohse M, Usadel B. Trimmomatic: A flexible trimmer for Illumina sequence data. Bioinformatics 2014:30:2114-2120.

19. Li H, Handsaker B, Wysoker A, Fennell T, Ruan J, et al. The sequence Alignment/map format and samtools. Bioinformatics 2009:25:2078-2079.

20. Comas I, Jaidip C, Peter MS, James G, Stefan N, et al. Human T cell epitopes of Mycobacterium tuberculosis are evolutionarily hyperconserved. Nat Genet 2010;42:498-503.

21. Virtanen P, Travis O, Matt H, Tyler R, David C, et al. SCIPY 1.0: Fundamental algorithms for scientific computing in Python. Nat Methods 2020;17:261-272.

22. Zignol M, Andrea C, Anna SD, Philippe G, Natavan A, et al. Genetic sequencing for surveillance of drug resistance in tuberculosis in highly endemic countries: A multi-country population-based surveillance study. Lancet Infect Dis 2018;18:675-683.

23. J A, Crampin AC, Houben RM, Mzembe T, Mallard K, et al. Largescale whole genome sequencing of $M$. Tuberculosis provides insights into transmission in a high prevalence area. Guerraassunção. elife 2015:4:e05166.

24. Forouzan E, Parvin S, Masoumeh SM, Karkhane AA, Yakhchali B, et al. Practical evaluation of 11 de novo assemblers in metagenome assembly. J Microbiol Met 2018;151:99-105.

25. Wajid B, Serpedin E. Review of general algorithmic features for genome assemblers for Next Generation sequencers. Genomics, Proteomics \& Bioinformatics 2012;10:58-73.

26. Goig GA, Silvia B, Alberto L, Garcia B, Iñaki C, et al. Contaminant dna in bacterial sequencing experiments is a major source of false genetic variability. BMC Biol 2020;18:1-15.
27. Cole S, Churcher C, Parkhill J, Garnier T, Harris D, et al. Deciphering the biology of Mycobacterium tuberculosis from the complete genome sequence. Nature 1998:396:190.

28. Chiner-Oms Á, Sánchez-Busó L, Corander J, Gagneux S, Harris SR, et al. Genomic determinants of speciation and spread of the Mycobacterium tuberculosis complex. Sci Adv 2019;5:eaaw3307.

29. Achtman M. Insights from genomic comparisons of genetically monomorphic bacterial pathogens. In: The Royal Society, 2012, Philosophical Transactions of the Royal Society B: Biological Sciences, Vol. 367. 2012. pp. 860-867.

30. Didelot X, Walker AS, Peto TE, Crook DW, Wilson DJ, et al. Withinhost evolution of bacterial pathogens. In: Nature Reviews Microbiology, Vol. 14. Nature Publishing Group, 2016. pp. 150-162.

31. Vos M, Didelot X. A comparison of homologous recombination rates in bacteria and archaea. ISME J 2009:3:199-208.

32. Didelot $X$, Wilson DJ. Clonalframeml: Efficient inference of recombination in whole bacterial genomes. PLoS Comput Biol 2015;11:e1004041.

33. Holley G, Melsted P. Bifrost - highly parallel construction and indexing of colored and compacted de Bruijn graphs. bioRxiv 2019.

34. Colman RE, Schupp JM, Hicks ND, Smith DE, Buchhagen JL, et al. Detection of low-level mixed-population drug resistance in Mycobacterium tuberculosis using high fidelity amplicon sequencing. PLoS One 2015;10:e0126626.

35. Katebi M. In: Pathogist: a Novel Method for Clustering Pathogen Isolates by Combining Multiple Genotyping Signals. Simon Fraser University, 2019.

36. Zabeti H. An Interpretable Classification Method for Predicting Drug Resistance in M. tuberculosis. Cold Spring Harbor Laboratory, bioRxiv, 2020.

\section{Five reasons to publish your next article with a Microbiology Society journal}

1. The Microbiology Society is a not-for-profit organization.

2. We offer fast and rigorous peer review - average time to first decision is 4-6 weeks.

3. Our journals have a global readership with subscriptions held in research institutions around the world.

4. $80 \%$ of our authors rate our submission process as 'excellent' or 'very good'.

5. Your article will be published on an interactive journal platform with advanced metrics.

Find out more and submit your article at microbiologyresearch.org. 